\title{
Thu Thiem new urban center Master plan: urban design in the direction of adapting to the natural and cultural environment
}

\author{
Phu Cuong Pham ${ }^{1, *}$, Thi Ngoc Lan Nguyen ${ }^{1}$, Huu Toan Phan ${ }^{1}$ and Nguyen Song Hoan \\ Nguyen ${ }^{1}$ \\ ${ }^{1}$ University of Architecture Ho Chi Minh City (UAH), Pasteur str., 196, Ho Chi Minh City, Vietnam
}

\begin{abstract}
The aim of this research is to analyze the Master plan of Thu Thiem new urban center of Ho Chi Minh City, showing the advantages of the project in adaptation to the natural environment and local cultural environment: to inherit and convey the characteristics of historical urban space to the new urban center, to inherit the principle of zoning and functional integration in land use, to maintain harmonious connection with the historic urban center, to arrange a "compact" plan causing no impact to the natural environment, to preserve and embellish the water environment. This research also addresses issues that need to be further researched in the implementation of the Thu Thiem master plan, to ensure the adaptability to the natural and cultural environment of the project which will be successfully applied in practice: to consolidate the characteristics of modernity of new urban architecture, to make the central square diverse and harmonious with human scale, to organize the underground space and height in accordance with the TOD development model, to study the diversity of cultures and local communities in order to plan a unique community.
\end{abstract}

\section{Introduction}

The aim of this research is to analyze the Master plan of Thu Thiem new urban center of Ho Chi Minh City, showing the advantages of the project in adaptation to the natural environment and local cultural environment.

Object of research: detailed Master plan 1/2000 scale of Thu Thiemnew urban center of Ho Chi Minh City, also known as Sasaki plan. The plan was implemented by Sasaki Associates (USA), and was approved by the People's Committee of Ho Chi Minh City in 2005.

\section{Literature review}

Urban development in Thu Thiem is a relatively new development compared to the history of 300 years of urban development of Saigon-Ho Chi Minh City.

\footnotetext{
* Corresponding author: cuongkientruc@yahoo.com
} 
Prior to the approval of the Sasaki project in 2005, the Thu Thiem area was researched and incorporated into planning plans such as the Hoang Hung plan; Doxiadis-USA (1968); WBE-USA (1972); Ho Chi Minh City Master Plan- Vietnam (1995) [6,7].

In 2003, Ho Chi Minh City organized the international competition for new planning concepts for Thu Thiem new center. One of the main requirements of the competition is that the plan must convey the characteristics of local culture into the planning of new urban center. The panel of judges, including John Lang (USA), William Lim (Singapore), Tran Du Lich (Vietnam) awarded the first prize to Sasaki. The winning plan had also been selected to develop into a detailed Master plan of 1/2000 of the new Thu Thiem urban center (Sasaki plan) $[2,3,10]$.

After Sasaki's plan was approved, the planning of Thu Thiem was compiled a book named Thu Thiem - Past and Future by Ton Nu Quynh Tran (2010). In this book, the analysis of the issue of adaptation to natural and cultural environments in Sasaki's Thu Thiem master plan was mentioned by Nguyen TrongHoa, Nguyen Minh Hoa and Nguyen QuangVinh $[4,5,8]$.

\section{Materials and methods of research}

Site of research: Thu Thiem is a peninsula located opposite to existing historic center of Ho Chi Minh City through Saigon river, very similar to the location of the Pudong district opposite to center of Shanghai City (China). Despite its special location, the development of Thu Thiem has always been overlooked during the urbanization of Saigon in the French colonial period (from 1860s to 1950s) due to its low-lying terrain concerns.

Since the 1950s, due to the need to expand Saigon, planners began to pay attention to Thu Thiem, and have included the region into many urban development plans. There are always differences in views on the scale of development. Hoang Hung plan proposed that Thu Thiemshould become a new center with a large development scale to replace the former administrative center of Saigon. The well-known Doxiadis planner proposed a pilot model of low-rise residential buildings intermixing the canal system to avoid interference to the terrain and geology of the area (The Saigon-Thu Thiemplan in 1968). WBE's plan of Thu Thiem1972 criticized the linear nature of Doxiadis as pushing the traffic flow across the Saigon River across the peninsula. This plan proposed to develop Thu Thiem into Southeast Asia's largest commercial-service center [6,7].

In 1995, on the basis of the masterplan of the city, Thu Thiem was identified as the new development center of Ho Chi Minh City. However, the economic changes in Asia made the project unattractive for investment.

In 2003, the demand for Ho Chi Minh City's expansion has once again placed Thu Thiem in a strategic position. The city has launched the international competition for new planning concepts for Thu Thiem new center with the participation of leading Vietnamese and international consultants. The proposed plan of Sasaki (United States) won the first prize and was selected to develop into the detailed Master plan of the new urban center of Thu Thiem.

In 2005, detailed Master plan of Thu Thiemnew urban area with a scale of 1/2000 (Sasaki project) was approved by the People's Committee of Ho Chi Minh City [2,3,10]. 


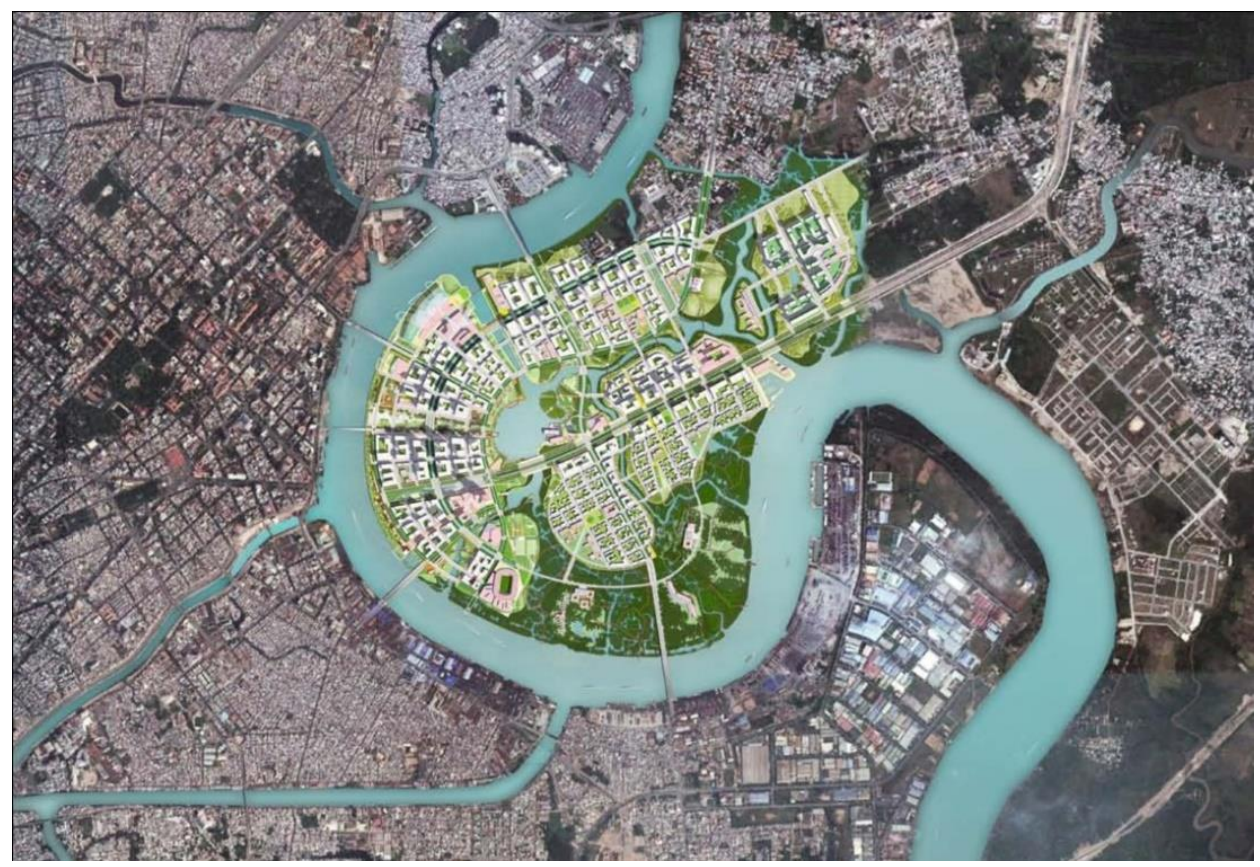

Fig. 1. Map comparison method (to analyze the relationship between urban layout between Thu Thiem and HCM historic center), historical and logical method, and Analysis- synthesissystematization.

\section{Results}

The prominent achievements of Sasaki's project in adapting to the natural environment and the local cultural environment:

- Inherit selectively, convey the typical values of the historic urban center to the new urban center space:

As an important element in the ongoing development of Saigon-Ho Chi Minh City, Thu Thiem new center is not a space in opposition to the existing center. It is relatively easy to build a modern urban space separate from the former city. The question is how to convey the cultural imprints and seamlessly connection to the existing historic center in the context of modernization of the new urban center.

The highlight of Sasaki's plan compared to others in Thu Thiem planning concept competition was that it conveyed the imprint of the historic urban space to the new center space. It is evident in the similarity of road network structure, street layout and layout of the construction works.

The structure of the road network of Thu Thiem center not only inherits the characteristics of the historic chessboard box network, but also shows a clearer adaptation to the nature. While the urban center of the existing center shows the domination of the geometry over the river, the Thu Thiem road network is a combination of chessboard road and curved harmonic to natural terrain.

The street block structure has similarities in scale to the existing center, creating a good sense of "human scale" on a concise and friendly scale. The high-density layout of the structure, along the road leading to the character of living communication for street space; the center of street blocklies a safe open space for community activities. 
The "compact" urban layout has contributed to the zoning of the built environment, retaining many of its natural surroundings and features, giving Thu Thiem a harmonious combination of built environment and natural environment rich in identity.

Highlights in functional layout are the combination of functional zoning principles and complex land use features. Complexity ensures the diversity and vitality of the city center, avoiding the creation of "dead" areas due to a lack of urban activity, demonstrating the traditional intangible cultural imprints of urban activity.

With its high density of "compact" development, diverse functions, chess board network, friendly street-rate, street-based interactive interfaces, the Sasaki plan inherits and conveys values of the historic city successfully.

To reaffirm the success of the Sasaki plan in conveying the existing urban features, let us quote the comment of panel of judges, which includes well-known experts such as Jon Lang and William Lim, "not an escape from the old city"; on the contrary, it "brought the imprints of the historic city through the river" [9].
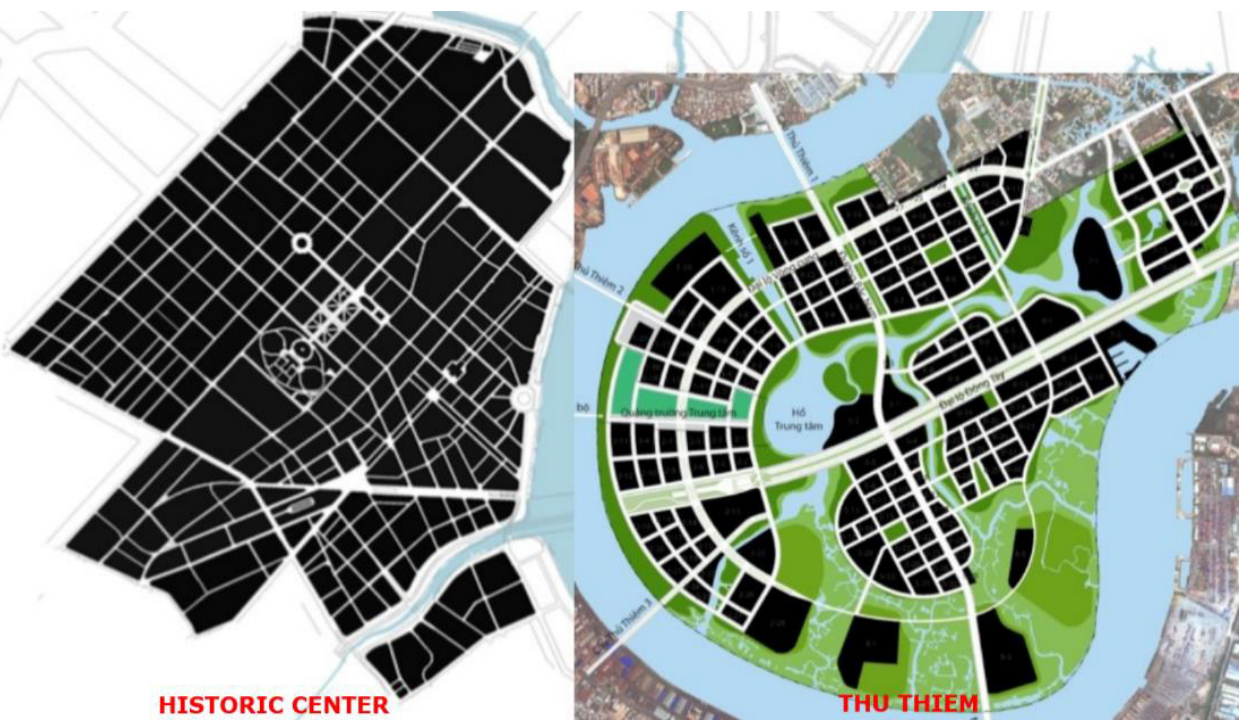

Fig. 2. Historic center and Thu Thiem.

- Transport and landscape spaces connecting with the existing center:

The history of urban development in Ho Chi Minh City reflects the general development law of estuarine cities, based on the advantages of river transport routes to accelerate the urbanization process. However, over the past 300 years, while the Saigon River has always been an invaluable support to the development of existing urban space, it is a natural object that limits the development of space towards the East bank. In that context, Thu Thiem - a land of great potential, has been almost forgotten during the development stages of the city. The Sasaki master plan has opened up a practical opportunity to bring urban development across the river, linking Thu Thiem with the Saigon River and the existing center, creating the final piece of the central space of Ho Chi Minh City [5].

With a special advantage of its location and abundant land for construction, Thu Thiem will receive various projects with modern functions, sharing the pressure of intense development in the current historic center.

The existing center has the condition to maintain the urban heritage values that represent the city's development in the nineteenth and twentieth centuries. Thu Thiembrings an opportunity to convey, connect traditional characteristics, and at the same time, adding 
functional and stylish works to mark the development of the 21 st century in a harmonious and continuous development process.

Regarding traffic, Thu Thiem is connected to the existing center by various types of traffic. Motorized traffic through five bridges and one tunnel, public transport by metro, waterway transport by large water transport vehicles and water taxi. In particular, the plan proposed a pedestrian bridge across the Saigon River linking the central square space to the existing Me Linh square.

Functionally, Thu Thiem is defined as an extension of the city center, adding new functions that the existing center has not been able to develop due to space limits. This is a twin and symbiosis city of the existing center, and develops that symbiotic relationship through international business and service oriented operations.

The plan proposed the planning of highlight buildings to mark new cultural values, such as international convention and exhibition center, museums, observation towers, trade finance centers and squares, etc. Views on urban landmarks have been studied from both the old and the new centers, creating a visual connection between the two river banks in Saigon. The most important viewpoint is formed from the central square, creating a seamless link to look at both old and new architectures.
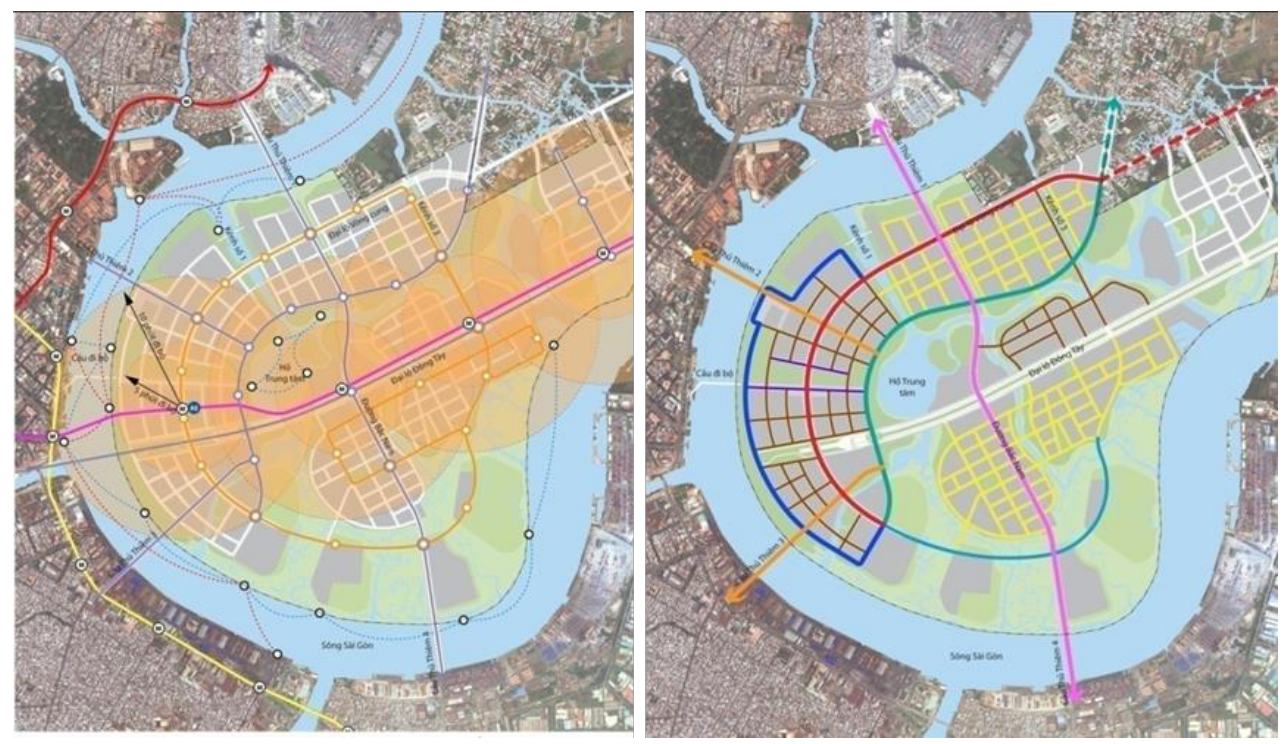

Fig. 3. The most important viewpoint from the central square, creating a seamless link to look at both old and new architectures.

- Preserve and embellish the identity of a river city:

The plan clearly demonstrates the idea of keeping a balance between development and environment protection and embellishment. Derived from the solution to increase urban density under the model of "compact" development, the plan concise planning space. Rather, the compact structure allows for the selective determination of areas that are leveled to develop the building. The area not leveled shall be preserved, protected and embellished in order to harmonize urban space with the natural environment of the area.

The idea has preserved a large area of the peninsula's delta space with its nearly intact nature. The plan has proposed environmentally-friendly planning solutions that ensure that natural features are not obscured or overlapped in the process of urban development. Specifically, the plain aims to protect and embellish existing canals and open large reservoirs to regulate water levels to avoid flooding and to open up the urban canal streams flowing from the SaiGonriver to the central lake. 
It is noteworthy that delta spaces are not only protected as an ecological element but also integrated into the plan to be developed into unique open spaces. The project interlocked the natural landscape with functional areas of the city, forming the main framework to shape the entire open space system of Thu Thiem center. These types of open space associated with the natural waterfront landscape such as riverbank park, urban canal, natural canal, central lake, boat landing, ecological forest will form a landscape system diverse in function. And more importantly, this format bring Thu Thiema uniqueness of a urban river in the South, conveying the cultural mark of urban "boat and dock" into modern urban life. .

While the river element has been significantly damaged in urban development in the existing center, the dynamic linking of the delta's natural signature to the urban space of Thu Thiem is an opportunity to consolidate strong identity, the uniquenessof the central HCMC compared to other cities, both domestically and internationally $[5,10]$.
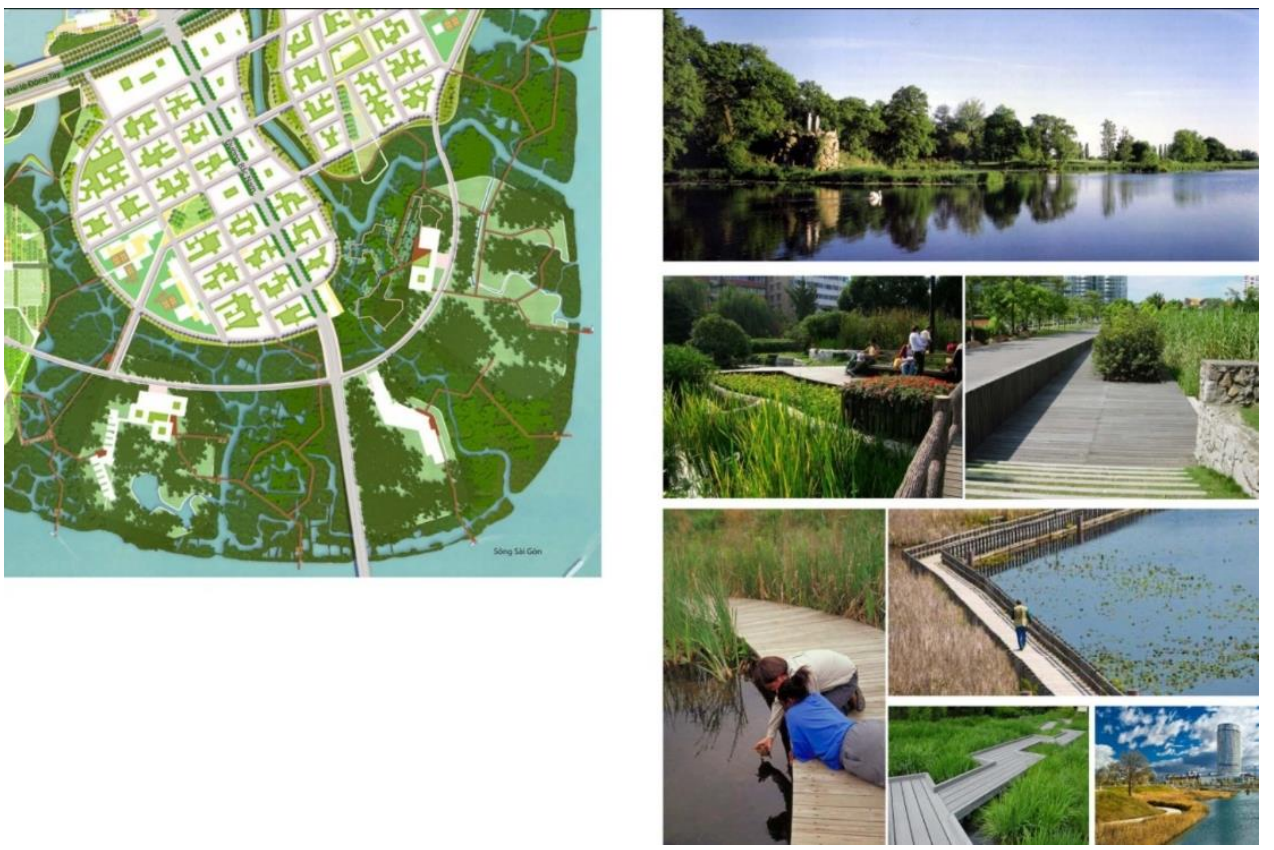

Fig. 4. The central HCMC.

\section{Discussion}

This research shows the great successes of the Sasaki plan in terms of adaptation to the natural environment and local cultural environment. In this part we will discuss issues that need to be further researched in the implementation of the Thu Thiem master plan, to ensure that adaptation to the natural and cultural environment of the plan that will be successfully applied in practice.

- Consolidate the modernity characteristics of new urban architecture:

If the existing center represents the development of the city in the nineteenth and twentieth centuries, Thu Thiem represents the new development of the center of Ho Chi Minh City in the 21 st century. That is why architecture in Thu Thiem must showcase the values of modernity in urban development.

Currently, the plan has not specifically mentioned the principles of determining characteristics of architectural form and style. This may lead to the risk of repeating the past lessons in developing new urban areas with architectural style that do not fully reflect the 
characteristics of the modernity. New "portraits" of urban style with a pasty, arbitrary, even asylum copy style like Putrajaya (Malaysia) or, to at other level, Ciputra (Hanoi) can be considered as high alert examples.

Therefore, in order to have an architectural appearance that represents the characteristics of the modernity and the locality, there should be specific regulations for the form of the building architecture. The architectural style at Thu Thiem should convey the value of diversity, but it should not copy the architectural forms of the former center. It should not mimic the Western ancient architecture style as it overlaps with the old urban architecture, blurring and even invoking the true values of the existing center. Architectural forms that are characterized by adaptability to the natural environment and in accordance with the modernity, technology and materials are the appropriate approaches to be encouraged.

For landscape architectural space planning, Sasaki's plan offers the principle of "expressing a city created by spaces connected by" axes "and" highlights" [10]. This principle is demonstrated by the layout solution of structures on important axial connection. However, due to the fact that most of the works identified as urban landmarks are of low-story scale, it only promotes the value of the axes, without creating a distinctive mark on the urban silhouette. In fact, as a large-scale urban development center, Thu Thiem's urban silhouette will be identified primarily through the layout of high-rise buildings rather than the low-story buildings.

The analysis of the planning sections of the Sasaki plan, the silhouette image is not lively. The reason is that because most of the high-rise buildings in the same planning area are of uniform height, the image of the urban horizon lacks the emphasis on height, which fails to create a rhythm.

Urban design therefore needs to propose a silhouette image with a clearer idea, through high-level re-distribution on the principle of diversity and flexibility. High-rise buildings should be organized in the form of transitions of height and affirm the highest points, instead of the uniform height without sudden change. The image quality of the urban cross-section along the banks of the Saigon River (opposite to Me Linh Square), the central square and the East-West Boulevard need to be improved to show a 21 st century urban image which is dynamic and modern [9].

- Enhance the communication and "human scale" of public spaces:

The largest central square in Vietnam with an area of up to 20 ha is a factor that needs to be considered. Well planned for large events with millions of participants, the operation of this public space at non-festive times may be influenced by the enormous size. Because the vitality of public space is shaped by the ability to communicate with the community, from human interaction with everyday events rather than its physical size. Lessons learned from the enormous but listless public spaces of many modern metropolises have been the basis from which many top urban designers propose reasonable limits for the square space, with the scale limited at the human rate.

Therefore, it is recommended to diversify the functions of the Thu Thiem central square. The solution is the layout of the square should be in the form of clusters of squares, open spaces of various sizes and functions instead of a large square of big size. Open spaces such as parks, waterways, lawns, outdoor stages serve better for the diverse needs of the community. The parameters needed for squares must be in line with human communication that are data used as reference in design [9].

- Organizational orientation of underground space and appropriate height for TOD development model:

With the "compact" development model of a new center, Thu Thiem needs a detailed underground development plan in advance. Organizing underground parking spaces and public and commercial activities, as well as pedestrian connectivity between buildings are effective for "decompression" measure for high density urban center. The reason is that it can 
release most of the ground area for open space, green area, maximize the pedestrian connection.

Derived from the advantages of a new development area, less affected by current technical conditions, Thu Thiem planning should clearly indicate the direction of underground space so that technical solutions can be researched, selected and implemented uniformly from the very first stage of development.

The specific solution is to study the connection of underground space for high-density areas such as the central core and the multi-functional East-West Highway area. The function of underground space is not just a parking lot, but it needs to be integrated with other diverse functions such as commercial space, public-cultural space, and pedestrian connection to buildings in the area and to the Metro station.

In addition, the distribution of architectural planning criteria of Thu Thiem should be further studied to be more suitable with the development trend of TOD. Land height and coefficient should be increased around the main Metro stations. In other words, the location of the Metro stations should be the areas with the highest coefficients to optimize its development potential [9].

- Study the diversity of culture and local communities at site:

In terms of nature, Thu Thiem planning is not a case of at-site upgrades, but the long-term resident community will be relocated to another place, to be able to develop a new space and community. This approach help urban planner and designer not be burdened by historical communities. However, in reality, even though its current status is a river with a tentative lifestyle, Thu Thiem still "contains very special cultural values, a combination between countryside and urbanity "[8]. There are still a number of religious construction works. These works, though small in number, are not large, but they contain manifestations of spiritual life, intangible cultural values associated with Thu Thiem.

In addition, the phenomenon of space separation and social polarization are stern warnings drawn from the practice of population organization in many new urban areas in Asia. These lessons allow us to recognize that the process of settling in Thu Thiemcan not be completely abandoned for the adjustment of the market $[1,8]$.

For that reason, in order to ensure that the plan does not fluctuate profoundly or even in the face of market pressures that lead to spatial separation and social polarization, the State should have positive intervention through social planning tools and policies that encourage diversification in investment in residential construction.

In the process, management and implementation of urban design on the one hand must protect the maximum urban configuration with the identity of the delta of the design plan. On the other hand, it should indicate the tools and policies needed to ensure that the urban population of Thu Thiem is structured and has equal quality, capable of engaging in the operation of an economic, financial and cultural apparatus of a modern city. The urban community must absolutely avoid being closed, and will be an open, balanced, harmonious society in harmony with the existing city community. Actively facilitating a suitable percentage of Thu Thiem residents to work and reside in the new urban area can be considered as a solution to enhance identity and social equity for urban communities of Thu Thiem in the future.

\section{Conclusions}

Opposite to the Saigon River, Thu Thiem has a close and symbiotic relationship with the existing historic center of Ho Chi Minh City. Therefore, Thu Thiem new urban center should not be isolated as a cultural break. On the contrary, it should be oriented to become a quality piece, showing both imprints of modernity and the values of historic urban architecture and preserve the identity of the local natural environment. 
This study identified the strengths of the detailed Master plan of Thu Thiem new urban center (Sasaki plan) in adaptation to the natural environment and local cultural environment:

- to inherit and convey the characteristics of historic urban space to the new urban center

- to inherit the principle of zoning and functional integration in land use

- to maintain harmonious connection with the historic urban center

- to arrange a "compact" plan, causing no impact to the natural environment

- to preserve and embellish the water environment

This research also addresses issues that need to be further researched in the implementation of the Thu Thiem master plan, to ensure the adaptability to the natural and cultural environment of the project which will be successfully applied in practice:

- to consolidate the characteristics of modernity of new urban architecture

- to make the diverse central square diverse and harmonious with human scale

- to organize the underground space and height in accordance with the TOD development model

- to study the diversity of cultures and local communities in order to plan a unique community

\section{References}

1. J. Lang, Urban Design: A Typology of Procedures and Products (Architectural Press, USA, 2005)

2. People's Committee of Ho Chi Minh City, Decision on approving the detailed plan of Thu Thiem new urban center with a scale of 1/2000 (Ho Chi Minh City, 2005)

3. People's Committee of Ho Chi Minh City, Decision on approving the detailed plan of urban construction 1/2000 scale (zoning plan) the existing center of HCM City (930ha) (HCMC, 2012)

4. Nguyen Minh Hoa, From the communication space to human space, Thu Thiem-past and future (General Publishing House, Ho Chi Minh City, 2006)

5. T. Nguyen, Conveyance of cultural characteristics of Saigon-Ho Chi Minh City in the area of planning Thu Thiem new urban area, Thu Thiem-past and future (General Publishing House, Ho Chi Minh City, 2010)

6. H. T. Nguyen, Contemporary Vietnamese Architecture Issues (Construction Publishing House, Hanoi, 2002)

7. H. T. Nguyen, Overview of city architecture, Ho Chi Minh City Cultural City, Volume III (Ho Chi Minh City General Publishing House, Ho Chi Minh City, 1987)

8. Q. V. Nguyen, The general forecast of Thu Thiem urban population in the future, Thu Thiem past and future (Ho Chi Minh City Publishing House, HCMC, 2010)

9. P. C. Pham, Maintaining and conveying typical urban architectural values in the context of expanding the existing center area of Ho Chi Minh City, Ph.D. thesis (2015)

10. Sasaki, Final Master Plan report for Thu Thiem new urban center (Hochi Minh City, Viet Nam, 2005) 